\title{
TOMÁS DE AQUINO SOBRE LA NATURALEZA DE LO MENTAL'
}

Jörg Alejandro Tellkamp**

SINTESIS - Sin lugar a duda, reflexiones sobre la naturaleza de la mente han preocupado a pensadores a lo largo de los siglos. Hoy en dia, la Filosofia de la Mente enfoca el fenómeno de lo mental principalmente desde un punto de vista fisicalista. Esto quiere decir que todos los procesos mentales de una $\mathrm{u}$ otra manera son descriptibles en términos de lo que ocurre en el cerebro a nivel neuronal. Parece que esta apreciación se aleja considerablemente del concepto de lo mental en la Edad Media. De esta manera parecería que en Tomás de Aquino lo mental queda relegado a una descripción dualista que afirma una diferencia esencial entre lo mental y lo corporal. Quisiera entonces, en esta comunicación, presentar una imagen un tanto distinta, según la cual los procesos mentales según el Aquinate no se limitan al intelecto. Se tendrá que incluir la teoría de los sentidos interiores, los que representan facultades cognitivas que llevan a los ser sensibles, y también a seres humanos, a reconocer su mundo circundante de una manera no trivial. Un análisis de esas capacidades mentales demostrará que son necesarios criterios fisicalistas para poder asir adecuadamente lo mental en la teoría de Tomás de Aquino.

PALABRAS-CLAVE - Tomás de Aquino. Naturaleza de la mente. Procesos perceptivos y emocionales. Intelecto y cuerpo.
ABSTRACT - Without place to doubt, reflections on the nature of the mind have worried thinkers along the centuries. Nowadays, the Philosophy of the Mind focuses the phenomenon of the mental thing mainly from a physicalist point of view. This means that all the mental processes in one or another way are descriptive in terms of what happens in the brain in the neuronal level. It seems that this appreciation moves considerably away from the concept of the mental thing in the Middle Age. This way it would seem that in Thomas Aquinas the mental thing is relegated to a dualistic description that it affirms an essential difference between the mental thing and the corporal thing. I'd want then, in this communication, to present an image a little different, according to which the mental processes according to Aquinas are not limited to the intellect. One will have to include the theory of the interior senses, those that represent abilities of knowledge that take to the sensitive being, and also to human beings, to recognize their surrounding world in a non trivial way. An analysis of those mental capacities will demonstrate that they are necessary physicalist approaches to be able to seize the mental thing appropriately in the Thomas Aquinas's theory. KEY WORDS - Thomas Aquinas. Nature of the mind. Mental processe. Intellect and body.

* Agradezco a Margarita Múnera y Juan Fernando Mejía por la ayuda que me brindaron en la redacción de esta ponencia. Evidentemente, los errores que todavia contiene, son de responsabilidad mía.

** Pontifícia Universidad Javeriana. 
La naturaleza de lo mental ha sido objeto de reflexiones a lo largo de los siglos, como lo vemos, por ejemplo, en Platón y Aristóteles, entre muchos otros. En la discusión actual, sobre todo en el mundo anglosajón, se destaca este tema en una rama de la filosofía contemporánea denominada Filosofía de la Mente. Su enfoque principal es de tipo fisicalista. Esto significa que todos los procesos mentales de una u otra manera se relacionan con el sustrato físico del cerebro. Todas las clases de computación y de procesos mentales son, según lo afirma la mayoría de los pensadores al respecto, reducibles a una descripción de lo que ocurre en el cerebro a nivel neuronal.

Parece que esta apreciación se aleja considerablemente del concepto de lo mental en la Antigüedad y la Edad Media. En Aristóteles, por ejemplo, vemos que el nous no precisa de órganos, y, de manera parecida, Agustín de Hipona relaciona el intellectus con una propiedad incorpórea del ser humano. Igualmente parece que en Tomás de Aquino lo mental queda relegado a una descripción fundamentalmente dualista que sostiene una diferencia esencial entre lo mental y lo corpóreo.

En mis anotaciones intentaré demostrar que la apreciación tradicional sobre lo mental en Tomás de Aquino tiene que ser sometida a una revisión. Por un lado no cabe la menor duda que la descripción tradicional es correcta en el sentido en que el intellectus y sus funciones se encuentran esencialmente independientes de factores orgánicos. Por el otro lado, y matizando esta afirmación, intentaré argumentar en favor de lo siguiente:

1. Una teoría sobre lo mental en el pensamiento de Tomás de Aquino no se agota en la teoría del intelecto.

2. Lo mental también incluye procesos perceptivos y emocionales racionalmente cargados que necesariamente se basan en un sustrato físico.

3. El dualismo intelecto-cuerpo, por lo tanto, no tiene que ser rechazado, sino que tiene que ser sometido a una revisión.

En primer lugar quisiera aclarar el concepto de lo mental que utilizaré a continuación. Cuando hablo aquí de "lo mental", se quiere marcar una diferencia con lo que en la tradición desde Agustín se llama mens. De esta manera, al referirme a lo mental utilizo una acepción contemporánea, la cual, por lo tanto, no se ajusta plenamente a la definición que Tomás da de mens como de una facultad independiente de un sustrato orgánico. ${ }^{1}$ De esta manera, quisiera hablar de lo mental en vez de la mente, refiriéndome a todas aquellas facultades y realizaciones cognitivas y desiderativas que un ser sensible y racional es capaz de realizar, es decir pensamientos, percepciones, deseos etc.

La psicología tomasiana tiene su fundamento en la metafísica. De manera general se puede constatar que en la discusión sobre lo mental en el siglo XIII los pensadores dominicos suelen tomar a Aristóteles como parámetro, en oposición a la mayoría de los franciscanos, cuyo parámetro es San Agustín. De modo que los conceptos fundamentales provienen de la metafísica aristotélica, sobre todo los

S.th. I q. 35 a. 5 c.: "[...] Mens non habet organum corporale”. 
que se refieren a la distinción entre materia y forma y, además, entre potencia y acto. Siguiendo al De Anima del Estagirita y utilizando los conceptos previamente mencionados, Tomás parte entonces de una serie de supuestos que tienen una incidencia directa sobre su concepto de lo mental, entre los que hay que destacar los siguientes:

a. Materia y forma son conceptos interdepebndientes.

b. El alma racional es la forma de un cuerpo que potencialmente tiene vida, en este caso del cuerpo humano.

C. El alma racional humana, por lo tanto, se da únicamente en cuerpos vivientes.

d. El alma intelectiva es acto puro.

e. Por lo tanto no depende esencialmente de un sustrato orgánico.

En los puntos mencionados evidentemente se debaten problemas intrínsecos a la caracterización de la relación entre el intelecto como forma corporis y el cuerpo mismo, pero no entraré en los detalles de esta discusión. Lo mental en Tomás no se refiere tanto a la constitución ontológica del ser humano en cuanto ser viviente, es decir al alma intelectiva, sino en primera instancia a su constitución cognitiva. Esto quiere decir que lo mental tiene relación con aquellas facultades, mediante las cuales los seres vivientes conocen sensorial o intelectivamente su mundo circundante.

El ser humano es un ser que, dado el caso que está informado por un alma intelectiva, tiene capacidades que Tomás llama potencias del alma (potentiae animae). ${ }^{2}$ ¿Qué es una potencia del alma? Como ya se ha dicho, el punto de parti$\mathrm{da}$, en lo que al tema propuesto se refiere, se encuentra en los conceptos de materia y forma. ${ }^{3}$ Por lo general, utiliza estas dos expresiones para indicar que una cosa compuesta está conformada de algo material y que esa cosa pertenece a una especie. La forma es aquello que designa la pertenencia a una especie, mientras que la materia representa el principio que individualiza esa cosa. ${ }^{4}$

Estrechamente relacionadas con los conceptos de forma y materia se encuentran las expresiones acto y potencia. ${ }^{5}$ Una cosa obtiene su determinación específica a través de la forma. La materia, en cambio, es aquello que es actualizable, lo que significa que de suyo la materia es indeterminada en cuanto a la obtención de una u otra forma. Forma y materia, y por lo tanto, potencia y acto, conforman la base para poder describir cualquier tipo de cambio en cosas. ${ }^{6}$

También la definición de lo que es una potencia del alma es incluida en la teoría de acto y potencia. Para que seres vivientes sean capaces de relacionarse activamente con el mundo exterior, tienen que tener la habilidad de sobrepasar

2 Cf. S.th. I q. 77

3 Véase como introducción a la terminología de forma y materia MEYER, Hans, Thomas von Aquin, Sein System und seine geistesgeschichtliche Stellung, Schöningh, Paderborn $2^{\mathrm{a}}$ edición 1961, p. 79101. Cf. también KenNy, Anthony, Aquinas, Oxford University Press, Oxford 1980, p. 32-60.

4 Cf. TOMAS DE AOUNO, De ente et essentia II, 36: "I...] Designatio speciei respectu generis est per formam, designatio autem individui respectu speciei est per materiam [...]".

5 Cf. MEYER op. cit. p. 79 ss.

B Cf. KeNNY op. cit. p. 38. 
sus limitaciones corporales. Las piedras, por ejemplo, no son capaces de relacionarse con el mundo exterior, justamente porque no poseen esta habilidad. Potentiae animae son, según Tomás, propiedades que le corresponden únicamente a los seres vivientes.'

En el contexto de la delimitación conceptual de lo que es una potencia del alma, Tomás distingue tres tipos de almas, diferencia que se justifica por la diferente complejidad que se encuentra en los seres vivientes (gradus vivendi). ${ }^{8}$ Las actividades del alma se pueden manifestar, de acuerdo a la teoría de las tres almas, de tres maneras: la vegetativa, la sensitiva y la intelectiva. ${ }^{9}$ Lo vegetativo cumple la función de la alimentación y el crecimiento, trascendiendo de esta manera las limitaciones del cuerpo en que se manifiesta. ${ }^{10}$ Esta transgressio evidentemente también se aplica a las potencias sensitivas e intelectivas. Las potencias intelectivas no precisan de órganos corporales para su realización, y por lo tanto se alejan más que cualquier otra potencia de lo corporal. ${ }^{11}$ Las potencias sensitivas son jerárquicamente inferiores al intelecto, y ellas necesitan de la buena disposición (recta dispositio) de los órganos respectivos para poder realizar las actividades que les son propias. ${ }^{12}$

Con el concepto de potentia animae, Tomás intenta aclarar cómo los seres animados son capaces de trascender los límites de su corporalidad natural, para poder relacionarse activa o pasivamente con el mundo externo. ${ }^{13}$ Evidentemente la capacidad de representar mentalmente el mundo es una de estas formas.

Para comprobar que se puede interpretar lo mental en Tomás de acuerdo a principios fisicalistas, no es necesario, al mismo tiempo, negar la subsistencia del intelecto. Las observaciones anteriores demuestran que Tomás concibe al intelecto como potencia cognitiva que comparte los rasgos esenciales del alma humana, es decir que es inmaterial, indivisible e inmortal. Hasta este punto el concepto de lo mental en el Aquinate se mueve dentro de los parámetros tradicionales. Mi tesis es, en cambio, que en la descripción ontológica y cognitiva del intelecto no se agota en una teoría de lo mental, porque esto significaría que únicamente el intelecto sería capaz de computar con base en representaciones. ${ }^{14}$

7 Cf. S.th. I .q 75 a. 1. El concepto de vita es discutido en SANCTI THOMAE DE AQUino, Opera Omnia iussu Leonis XIII P.M. edita, Sentencia Libri De Anima, tomus XLV,1, Commissio Leonina/J. Vrin, Roma/París 1984, II, 1 p. 70.

s Cf. S.th. I q. 78 a. 1 c. También MEYER op. cit. p. 201s.

9 Cf. In DA II, 1 p. 71 s (ed. Leonina). Véase tambièn S.th. I q. 75 a. 1 c.

10 Cf. S.th. I q. 78 a. 1 c.: "Infima autem operationum animae est, quae fit per organum corporeum, et virtute corporeae qualitatis. Supergreditu tamen operationem naturae corporae, quia motiones corporum sunt ab exteriori principio, huiusmodi autem operationes sunt a principio intrinseco [...]. Et talis est operatio animae vegetabilis: digestio enim, et ea quae consequuntur [...]".

11 Ibid.: "Est ergo quaedam operatio animae, quae intantum excedit naturam corpoream, quod neque etiam exercetur per organum corporale. Et talis est operatio animae rationalis".

12 Ibid.: "Est autem alia operatio animae infra istam, quae quidem fit per organum corporale, non tamen per aliquam corpoream qualitatem. Et talis est operatio animae sensibilis [...]".

13 Cf. SANCTI ThOMAe De AouIno, Opera Omnia iussu Leonis XIII P.M. edita, Sentencia libri De Sensu et Sensato cuius secundus Tractatus est De Memoria et Reminiscencia, tomus XIV, 2, Commissio Leonina/J. Vrin, Roma/Paris 1985, cap. 1 p. 12, 52-54: "Virtus autem sensitiua, que inest animalibus, est quidem capax extrinsecorum, set in singulari tantum [...]".

14 Véase también TELL.LAMP, Jörg Alejandro, Sinne, Gegenstände und Sensibilia, Zur Wahmehmungstheorie des Thomas von Aquin, Brill, Leiden 1999, p. 218-289. 
En los escritos de Tomás de Aquino queda claro que los seres humanos tienen varias maneras de representar mentalmente al mundo sensible y cognoscible. Por un lado tenemos, como ya se ha visto, el contenido del conocimiento intelectivo, es decir lo inteligido. Esta clase de representación mental contiene, según Tomás, rasgos universales o sea géneros o especies. Sin embargo, el concepto de representación mental sobrepasa en cierta manera el conocimiento intelectivo, ya que también el conocimiento sensitivo se basa en representaciones mentales individuales. El conocimiento de lo que Tomás llama las intenciones individuales es alcanzado por una compleja organización de facultades mentales, las que denomina, en consonancia con la tradición, sentidos interiores (sensus interiores).

Los sentidos interiores son facultades mentales que tienen una ubicación marcadamente física en el cerebro. ${ }^{15}$ Además, tienen poderes causales, sobre todo en cuanto al conocimiento de cosas particulares. Es entonces importante reconocer que, según Tomás, lo que ocurre "en la cabeza" sí tiene relevancia respecto de la dimensión cognitiva tanto de los animales como de los seres humanos. Hay que destacar la causalidad intrinseca de los sentidos interiores respecto del conocimiento y la conducta, ya que en las últimas décadas se han dado ataques en contra de semejante apreciación, sobre todo por parte de Anthony Kenny. Según Kenny la teoría de los sentidos interiores en Tomás es incoherente. ${ }^{16}$ Los estados mentales no pueden ser episodios que se describen en términos causales, ya que esto conllevaría el peligro de tener que asumir un dualismo de sustancias. Lo mental, en cambio, se manifiesta más bien en forma de disposiciones adquiridas, las que no tienen efecto causal alguno sobre los sucesos observables. ${ }^{17}$ De esta manera, para Kenny una teoría causal del sensorio interior es un disparate conceptual.

Él declara que no es comprensible lo que significa percibir algo "interiormente", ya que este vocabulario es un claro indicio del ya caduco dualismo de mente y cuerpo. ${ }^{18}$ La doctrina de facultades mentales con poderes causales se basa en el error de aplicar la misma terminología a cosas de clases ontológicamente distintas, es decir a sustancias corpóreas y sustancias espirituales. Mientras que las cosas materiales se someten a las leyes de la mecánica, las sustancias espirituales se someten a leyes paramecánicas que operan con los mismos conceptos, pero aplicándolos a una esfera ontológicamente distinta ${ }^{19}$ Este procedimiento lleva a lo que Ryle denomina un error categorial.

Adicionalmente, Kenny amonesta que la teoría de los sentidos interiores no explica la existencia de facultades que superan la pasividad de lo meramente perceptivo. De esta manera la imaginación creativa no se incluye en tal teoría, y,

15 En este contexto prefiero hablar de facultades y no potencias, ya que únicamente potencias son individualizadas por un objeto propio, lo que no es el caso en los sentidos interiores.

16 Véase por ejemplo KeNNY, Anthony, Aquinas on Mind, Routledge, London 1993, p. 39: “Aquinas' treatment of the inner senses is not one of the more satisfactory parts of his philosophy of mind. In my view, the whole notion of 'inner sense' is misleading".

Cf. KENNY, Anthony, The Metaphysics of Mind, Oxford University Press, Oxford 1989, p. v-ix.

${ }^{18}$ Este es un reflejo del conductismo lógico; cf. RYLE, Gilbert, The Concept of Mind, Penguin, Harmondsworth 1990, p. 36-40.

19 Véase RYLE, op. cit. p. 20ss. 
por lo tanto, no es capaz de explicar lo que la tradición tomasiana y cartesiana pretende. ${ }^{20}$ Los eventos mentales no son, según Kenny, observables y consecuentemente su causalidad no es demostrable. ${ }^{21}$.

A mi manera de ver, esta crítica está errada por varias razones. Primeramente, no se puede pretender que teorías medievales, que en su mayoría adoptan esquemas causales de la mente, puedan ser interpretadas de manera conductista. Al desarrollar su teoría de los sentidos interiores y sus poderes causales, Tomás no disponia de la alternativa teórica del conductismo. Adicionalmente se puede constatar que en la discusión actual, el conductismo mismo ha sido descartado como alternativa para explicar lo mental. ${ }^{22}$

Para poder presentar una imagen menos sesgada que la de Kenny se tendrá que partir de los siguientes supuestos: (1) La referencia a sentidos interiores es razonable, para lo cual se pueden nombrar dos argumentos. Primero, esta teoría ayuda a explicar procesos cognitivos, como por ejemplo, la percepción. Segundo, la explicación de lo mental se basa en una teoría causal coherente. (2) El segundo supuesto es de naturaleza implícita. La relación entre las facultades perceptivas interiores y el sustrato orgánico permite establecer una identidad de estos dos ámbitos.

Ya se ha mencionado que tradicionalmente la relación entre propiedades mentales y corpóreas son interpretadas a la luz de la dicotomía entre la inmaterialidad del intelecto y la materialidad del cuerpo. El dualismo de estos dos elementos se justifica por el hecho que las propiedades del intelecto no pueden ser reducidas de ninguna manera a propiedades del cuerpo. La subsistencia del intelecto implica que sus actividades no precisan de manera esencial de factores orgáni$\cos ^{23} \mathrm{El}$ alma intelectiva y el cuerpo son, en principio, dos aspectos separables de un solo compuesto. ${ }^{24}$ Además de la distinción ontológica entre propiedades intelectivas y corpóreas, existe una razón epistemológica para separar estas dos esferas: si el intelecto fuese de naturaleza material, entonces no podría, debido a su limitación material, conocer todas las sustancias corpóreas. ${ }^{25}$ La indeterminación del intelecto respecto de su objeto propio es, en cambio, un atributo esencial suyo. El dualismo aquí esbozado reduce un tanto la complejidad del asunto, sin embargo, el punto central consiste en afirmar la incompatibilidad de atributos de lo intelectivo y lo corpóreo.

20 Cf. KENNY, Aquinas on Mind p. 40: "This is something which it would be more obviously absurd to call an 'inner sense'."

${ }^{21}$ Cf. RYLE, op. cit. cap. 1.

22 La reivindicación del concepto de la causalidad mental efectivamente llevó a la eliminación del conductismo lógico. Una descripción panorámica sobre teorias causales de la mente en JACKSON, Frank, "Mental Causation", en Mind 105 (1996), p. 377-413.

23

S.th. I q. 78 a. 1 c.: "Est ergo quaedam operatio animae, quae intantum excedit naturam corpoream, quod neque etiam execetur per organum corporale. Et talis est operatio animae rationalis".

24 Véase S.th. I q. 76 a. 1.

${ }^{25}$ Cf. S.th. I q. 75 a. 2 c.: "[...] Necessarium est dicere id quod est principium intellectualis operationis, quod dicimus animam hominis, esse quoddam principium incorporeum et subsistens. [...] Si igitur principium intellectuale haberet in se naturam alicuius corporis, non posset omnia corpora cognoscere". 
Los sentidos interiores representan formas de hablar sobre lo mental. A diferencia del intelecto, estos están esencialmente relacionados con un sustrato material, y esto significa que se manifiestan en determinados órganos. Por eso, la falta de un órgano específico implica la falta de la facultad interna correspondiente. De esta manera los sentidos interiores se dan únicamente en un sustrato orgánico, y, por lo tanto, no existe entre ellos y lo material una brecha ontológica como la que se constató en el caso del intelecto y su relación con el cuerpo. Por esta razón, la teoría de los sentidos interiores no puede ser interpretada dentro de los parámetros dualistas anteriormente mencionados. Parece que es entonces más plausible relacionar esta doctrina con una teoría de la identidad entre cuerpo y mente. ${ }^{26}$

De nuevo, lo que justifica esta identidad es la dependencia mutua entre el órgano y su correspondiente facultad. Cuando un órgano determinado no funciona plenamente, no es posible la actividad del respectivo sentido interior, lo que tiene repercusiones sobre la integridad del proceso cognitivo como tal. ${ }^{27}$ En este contexto hay que tomar en cuenta que la existencia del órgano se debe a la existencia de la facultad y vice versa. ${ }^{28}$ En esta dependencia mutua de órgano y facultad se pueden ver similitudes con teorías actuales de la identidad entre lo mental y lo material, y esto significa que Tomás estaría adoptando un punto de vista fisicalista respecto de algunos aspectos de la mente. ${ }^{29}$

Quisiera ahora, después de haber descrito las bases para una teoría de los sentidos interiores como facultades mentales, señalar un ejemplo de cómo se manifiesta esta teoría en un caso concreto. Para ello me referiré básicamente a la facultad interna de la vis cogitativa. Esta facultad es de especial interés, porque en ella se encuentra un punto de unión entre las potencias intelectivas y las facultades perceptivas en el ámbito de lo estrictamente físico.

Existe una larga tradición en lo que a dicha unión de lo mental y lo físico se refiere. Vemos, por ejemplo, en la obra de Nemesio de Emesa una facultad mental, el excogitativum, que tiene las propiedades de ser física y poder realizar actividades racionales. ${ }^{30}$ También en los siglos XI y XII se insinua una potencia mental

${ }^{26}$ La posibilidad de una identidad de facultades mentales y el sustrato físico se discute en RUNGGALDIER, Edmund, "Sentencia Libri De Anima und die modernen Identitätstheorien", en Lobato, Abelardo (ed.), L'anima nell'antropologia di S. Tommaso d'Aquino, Milano 1987, p. 605 - 613. Runggaldier limita su discusión al intelecto y no debate la posibilidad de facultades mentales orgánicas. Por lo tanto rechaza el autor una identidad de lo mental y lo corpóreo.

${ }^{27}$ S.th. I q. 84 a. 7 c.: "Videmus enim quod, impedito actu virtutis imaginativae per laesionem organi, ut in phreneticis; et similiter impedito actu memorativae virtutis, ut in lethargicis; impeditur homo ab intelligendo in actu etiam ea quorum scientiam praeaccepit".

Cf. S.th. I q. 78 a. 3 c.: "Non enim potentiae sunt propter organa, sed organa propter potentias [...]". Si en Tomás se puede hablar de una identidad, no queda claro cuál, si una identidad de tipos (types) o de realizaciones concretas (tokens). Sobre este tema véase ROSENTHAL, David M., "Identity Theories", en Guttenplan, Samuel (en.), A Companion to the Philosophy of Mind, Blackwell, Oxford 1994, p. 348-355.

Cf. NEMESIO DE EMESA, De Natura Hominis, Traduction de Burgundio de Pise, Verbeke, Gérard y J.R. Moncho (ed.), Brill, Leiden 1975 (= Corpus Latinum in Aristotelem Graecorum Suppl. 1), c. XI, p. 86, 59-67: "[...] Excogitativi autem sunt generaliter quidem iudicationes et depositiones et fugae et impetus actus, specialiter vero intelligentiae intelligibilium et virtutes et disciplinae et artium rationes et consiliativum et electivum [...]". 
física que corresponde a lo que a partir del siglo XIII se llamaba vis cogitativa. Para nombrar un ejemplo, en Guillermo de Conches encontramos el término rationalis, el cual coincide con el de la vis cogitativa, dado que ambos conceptos describen las mismas funciones y señalan al mismo espacio físico, es decir, a la mitad de la cabeza. ${ }^{31}$ Mientras que en siglo XII varía considerablemente la terminología respecto de esta facultad, en el siglo XIII comienza a imponerse un cierto estándar conceptual, sobre todo a partir de la lectura de las traducciones de Avicena y Averroes.

En todos los autores que se pronunciaron sobre este tema parece existir un consenso sobre que los seres humanos tienen (a) facultades sensitivas y que tienen (b) una facultad sensitiva que está marcada por procesos racionales, sin ser ella misma esencialmente racional y, por lo tanto, inmaterial. Tomás de Aquino aclara que esta facultad tiene efectivamente un locus orgánico:

"De este modo, lo que en los otros animales es llamada facultad estimativa natural, en el hombre es llamada cogitativa, porque descubre dichas intenciones por comparación. Por eso, es llamada también razón particular, a la que los médicos le asignan un determinado órgano que es la parte media de la cabeza, y, así, compara las intenciones particulares como la facultad intelectiva compara las universales." ${ }^{32}$

En oposición a la vis aestimativa, que se encuentra en los animales perfectos, la vis cogitativa cumple la función de reunir y comparar (colligere) las intenciones individuales, siendo éstas los signos complejos que representan un objeto particular. Los verbos colligere, inquirere y confere son utilizados para describir la actividad de la vis cogitativa. De esta manera se sugiere una actividad cuasi racional en el ámbito de lo orgánico. Esta actividad, sin embargo, no puede ser identificada con la de la razón misma, ya que no implica la aplicación consciente de conceptos y proposiciones, sino más bien la realización inconsciente de un conocimiento latente que John Searle llama background. ${ }^{33}$ De esta manera, es decir al poder distinguir y comparar intenciones individuales con base en un conocimiento racional latente, Tomás convincentemente argumenta a favor de facultades mentales que se manifiestan en el ámbito de lo orgánico.

Para concluir queda por recalcar que es imposible hacer una lectura facilista del problema de lo mental en Tomás. ¿Es él un dualista? La respuesta es: si y no. Si porque, por un lado, él afirma que el intelecto, sin duda una facultad mental, es separable en principio del sustrato orgánico. Por el otro lado, lo mental se manifi-

31 Cf. GUILLERMO DE CONCHES, De philosophia mundi IX, 24, col. 95 (PL 172): "Media [cellula-J.A.T.]

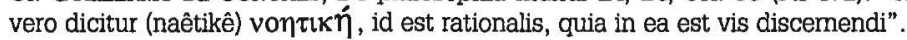

32 S.th. I q. 78 a. 4 c.: "Et ideo quae in aliis animalibus dicitur aestimativa naturalis, in homine dicitur cogitativa, quae per collationem quandam huiusmodi intentiones adinvenit. Unde etiam dicitur ratio particularis, cui medici assignant determinatum organum, scilicet mediam partem capitis, est enim collativa intentionum individualium, sicut ratio intellectiva intentionum universalium". La traducción procede de SANTO TOMÁs DE AQUino, Suma de Teología, Parte I, Biblioteca de Autores Cristianos, Madrid, 1988.

33

Cf. SEARLE, John R., Intentionality, An Essay in the Philosophy of Mind, Cambridge University Press, Cambridge, 1983 , p. $37-78$ y p. 141-159. 
esta, como se ha visto, en los sentidos interiores. El caso examinado, la facultad de la vis cogitativa, demuestra que existen actividades mentales intrínsecamente relacionadas con el sustrato físico que cumplen la función de darle al ser humano la posibilidad de conocer y reaccionar de una manera cuasi racional a los estímulos del mundo exterior. A mi parecer resulta entonces interesante ver que en un autor medieval como Tomás de Aquino se debaten problemas acerca de la naturaleza de lo mental de una manera muy semejante a la de las discusiones actuales. Es de esperarse que los expertos retomen el examen de la teońa de los sentidos interiores como una parte importante de la historia de la Filosofia de la Mente. 